Patients (or Participants) Twelve hundred and five athletes (86 teams) and 799 female athletes (78 teams), aged between 18 and 35 , participated to this study.

Interventions (or Assessment of Risk Factors) Survey data of athletes were collected twice during the season 2014-2015 (male athletes) and season 2015-2016 (female athletes).

Main Outcome Measurements Using an online survey (www. letselpreventie.be), information concerning type, duration and frequency of implemented exercises or barriers to neuromuscular training during the preseason and season were gathered.

Results During the preseason, only $12 \%$ of the athletes indicated to perform balance exercises (jump-landing) and plyometric exercises with a frequency of two sessions per week and a duration of at least 10 minutes per exercise. During the season, this percentage was reduced to $4 \%$. Fifty-five to $77 \%$ (preseason) and 65 to $82 \%$ (season) of the athletes respectively indicate not to perform any neuromuscular training at all. Most important barriers to neuromuscular training were: 'I don't need it', 'I have no time', 'stretching and warming-up are sufficient' and 'I don't know which exercises to perform'.

Conclusions Most non-elite soccer teams do not implement neuromuscular training for preventing ACL injuries. These results highlight the need for educating coaches and athletes to implement neuromuscular training during practice.

\section{IMPLEMENTING A SCHOOL PREVENTION PROGRAM TO REDUCE INJURIES THROUGH NEUROMUSCULAR TRAINING (ISPRINT): A CLUSTER-RANDOMIZED CONTROLLED TRIAL}

${ }^{1}$ Carla van den Berg, 1,23,4,5,7 Catyn Emery, 1,8 Sarah Richmond, 1,3 Derflingher, ${ }^{9}$ Carly McKay, ${ }^{1,5}$ Patricia K Doyle-Baker, ${ }^{1,10}$ Megan McKinlay, ${ }^{1,4,6}$ Clodagh Toomey, ${ }^{1,2,3,4,5}$ Alberto Nettel-Aguirre, ${ }^{11}$ Evert Verhagen, ${ }^{12}$ Kathy Belton, ${ }^{1,13}$ Alison K MacPherson, ${ }^{1,2,3,4,5}$ Brent Hagel. 'Sport Injury Prevention Research Centre, Faculty of Kinesiology, University of Calgary, Calgary, Canada; ${ }^{2}$ Department of Pediatrics, Cumming School of Medicine, University of Calgary, Calgary, Canada; ${ }^{3}$ Department of Community Health Sciences, Faculty of Medicine, University of Calgary, Calgary, Canada; ${ }^{4}$ Alberta Children's Hospital Research Institute, University of Calgary, Calgary, Canada; ${ }^{5} O^{\prime}$ Brien Institute for Public Health, University of Calgary, Calgary, Canada; ${ }^{6}$ McCaig Institute for Bone and Joint Health, University of Calgary, Calgary, Canada; ${ }^{7}$ Hotchkiss Brain Institute, University of Calgary, Calgary, Canada; ${ }^{8}$ Public Health Ontario, Health Promotion, Chronic Disease and Injury Prevention, Calgary, Canada; ${ }^{9}$ Department for Health, University of Bath, Bath, UK; ${ }^{10}$ Ever Active Schools, Calgary, Canada; ${ }^{11}$ Amsterdam Collaboration on Health and Safety in Sports, Department of Public and Occupational Health, Amsterdam UMC, Amsterdam, Netherlands; ${ }^{12}$ School of Public Health, University of Alberta, Edmonton, Canada; ${ }^{13}$ Department of Health Sciences, York University, Toronto, Canada

\subsection{6/bjsports-2021-I0C.48}

Background The effectiveness of neuromuscular training (NMT) programs in preventing injuries in youth sport is well documented, however there is little evidence on NMT programs delivered in school physical education (PE).

Objective To assess the effectiveness of iSPRINT, a NMT warm-up implemented in $\mathrm{PE}$ classes in reducing injuries and improving performance.

Design Cluster-randomized controlled trial.

Setting Junior high schools in Calgary, Canada.

Patients (or Participants) 1,067 students (12 schools; ages 1116 ; $53.7 \%$ female).

Interventions (or Assessment of Risk Factors) Following a workshop, teachers delivered a 12-week NMT (6 schools) or standard-of-practice (6 schools) warm-up at the beginning of PE classes.

Main Outcome Measurements Validated injury surveillance included sport/recreational injuries resulting in time loss from activity or medical attention. Predicted- $\mathrm{VO}_{2} \mathrm{max}$, vertical jump, and single-leg eyes-closed dynamic balance on foam pad were measured at baseline and 12-weeks. Multiple multilevel regression analyses (adjusting for previous injury and random effect by school and class) estimated injury incidence rate ratios (IRR) (Poisson regression considering interaction with sex) and mean changes in performance (linear regression).

Results iSPRINT was protective against all injuries $(\mathrm{IRR}=0.543$, 95\%CI; 0.295-0.998), lower extremity injuries (IRR $=0.357,95 \% \mathrm{CI} ; 0.159-0.799)$ and medically-treated injuries $(\mathrm{IRR}=0.289,95 \% \mathrm{CI} ; 0.135-0.619)$ in girls but not boys $\quad(\mathrm{IRR}=0.866,95 \% \mathrm{CI} ; 0.425-1.766,1.055,95 \% \mathrm{CI}$; $0.404-2.753$, and $0.639,95 \% \mathrm{CI} ; 0.266-1.532$; respectively). Mean baseline balance times (seconds) were similar between iSPRINT (7.4, SD+/-2.6) and control participants (6.9, SD + -2.2). At 12-weeks mean balance time was greater in iSPRINT group (9.1 SD+/-2.6) than control (7.9, SD+/-6.5). The difference in mean change over 12-weeks favoured the iSPRINT group (1.2 seconds, 95\%CI; 0.2-2.1). No between group differences were observed for changes in predicted$\mathrm{VO}_{2}$ max or vertical jump.

Conclusions An NMT program is effective in reducing injuries in girls and improving dynamic balance in all youth. This research informs the current standard-of-practice warm-up in youth PE. Future research should consider exercise fidelity differences between girls and boys.

\section{KINEMATIC AND NEUROMUSCULAR PREDICTORS OF FAILED LANDINGS DURING UNANTICIPATED DROP- JUMPS: IMPLICATIONS FOR INJURY PREVENTION}

${ }^{1}$ Nicholas Romanchuk, ${ }^{3}$ Céline Girard, ${ }^{1,2,3}$ Daniel Benoit. ${ }^{1}$ School of Human Kinetics, University of Ottawa, Ottawa, Canada; ${ }^{2}$ School of Rehabilitation Sciences, University of Ottawa, Ottawa, Canada; ${ }^{3}$ Ottawa Carleton Institute for Biomedical Engineering, Ottawa, Canada

\subsection{6/bjsports-2021-IOC.49}

Background Despite the link between jump landings and ACL injury risk, limited research has investigated the biomechanical and neuromuscular differences between successful and failed landings. Identifying the factors that lead to failed landings may provide specific targets for injury prevention programs.

Objective Identify which neuromuscular and biomechanical variables predict success or failure during unanticipated dropjump landings.

Design Cross-sectional study.

Setting Controlled laboratory setting.

Patients (or Participants) Thirty-two healthy male $(n=16 ; 15.9$ \pm 1.87 yrs.) and female $(15.7 \pm 1.7$ yrs.) Ottawa area competitive athletes with no history of major musculoskeletal injury affecting functional performance.

Interventions (or Assessment of Risk Factors) Participants completed single-leg drop-jump landings from a platform aligned to their tibial plateau. The landing leg was randomly signalled during flight via a projector in front of the participant. The landings were then categorized as 'successful' or 'failed' (defined as any loss of balance forcing the participant to adjust their base of support during landing). 